\title{
Gesamtinhaltsverzeichnis des Bandes 72 [1990]
}

Peter Blaho (Bratislava), Die Gestaltung des römischen Privatrechts . . . 234

RaJko Bratož (Ljubljana), Die Geschichte des frühen Christentums im Gebiet zwischen Sirmium und Aquileia im Licht der neueren Forschungen . . . . 508

Goča RevazoviČ Cecchladze (Moskau), Zu den kolchischen Sklaven in der griechischen Welt. . . . . . . . . . . . . . . . . . . .

HaNs Dixten (Berlin), Zu den Maũpor der Vita S. Pauli Junioris in monte Latro (10. Jh.), dem Personennamen Mauros (um 700), der Maupovøı́́o der Vita S. Petri Atroënsis und den Äthiopiern der Vita SS. 42 martyrum Amorien$\operatorname{sium}(9 . \mathrm{Jh}$.$) . . . . . . . . . . . . . . . . . . . . . . . . . .$

Erdka Endesfelder (Berlin), Noch einmal zum Feudalismus im Alten Ägypten 254

F. Javier Gómez Espelosín (Alcala de Henares), Oportunismo y demagogia. Los ecos de una reforma frustrade en Etolia. . . . . . . . . . . . . . .

Klaus Hallof (Berlin), Der Verkauf konfiszierten Vermögens vor den Poleten in Athen . . . . . . . . . . . . . . . . . . . . . . . . . . . . . 402

LÁszló Havas (Debrecen), Schemata und Wahrheit in der Darstellung der spätrepublikanischen politischen Ereignisse. . . . . . . . . . 216

Liselot Huchthauser (Rostock), Vergleich und Analogieschluß bei der Beurteilung von Sachverhalten in antiken Rechtsfällen. . . . . . . . . . . .

HeLGA KöPsterN (Berlin), Karl Krumbacher (23. 9. 1856-12. 12. 1909). - Mit einem Anhang: Zu zwei Briefen Karl Krumbachers an August Reifferscheid

BARBARA Kühnert (Jena), Zur sozialen Mobilität in der späten römischen Republik: plebs und ordo equester. . . . . . . . . . . . . . . . . .

Marek Kuryeowicz (Lublin), Das römische Recht und die Grundprobleme der Rechtsvergleichung . . . . . . . . . . . . . . . . .

Jon Edward Lendon (New Haven/Connecticut), The Face on the Coins and Inflation in Roman Egypt . . . . . . . . . . . . . . . . 106

Tadeusz Eoposzko (Lublin) - Henryk Kowalski (Lublin), Catilina und Clodius - Analogien und Differenzen . . . . . . . . . . . . .

Detref Lotze (Jena), Spielräume sozialer Mobilität in Athen und Sparta. Überlegungen zu verschiedenen Ausprägungen griechischer Gesellschaft in klassischer Zeit . . . . . . . . . . . . . . . . . . . . . . 
Christian Mileta (Berlin), Zur Vorgeschichte und Entstehung der Gerichtsbezirke der Provinz Asia . . . . . . . . . . . . . . . . . 427

Peter Panttscher (Graz), Zur Darstellung der Alexander- und Achaemenidennachfolge als politische Programme in kaiserzeitlichen Quellen. . . . . .

Maria Papathanassiou (Athen), Astronomie, Astrologie und Physik in der Rede Kaiser Julians auf König Helios. . . . . . . . . . . . . . . . . . . 498

$J_{A N}$ Pečírka (Prag), Athenische Demokratie und Fortschrittsdenken gegen Ende des 5. Jahrhunderts v. u. Z. : eine falsche Analogie. . . . . . . . . . .

GARY REGER (Hartford, Conn.), Some Remarks on "IG XII 8, 262 complété» and the Restoration of Thasian Democracy . . . . . . . .

Bernhard Rink (Berlin), Diskussionsbemerkungen zu dem Aufsatz „Catilina und Clodius - Analogien und Differenzen" von T. Koposzko und H. Kowalski ..................... 2 211

TAdeusz Sarnowskr (Warschau) - Piotr Dyczek (Warschau), Novae in 1987 West Sector. Results of the Polish Archaeological Expedition . . . . . . 173

WALTER ScheIDEL (Wien), Quasikolonen bei Vergil?. . . . . . . . . 166

EDITH SchöNERT-GEISs (Berlin), Das moesisch-thrakische Währungssystem in der römischen Kaiserzeit . . . . . . . . . . . . . . . . . . .

PaUL SpeCK (Berlin), Ikonen unter dem Kopfkissen oder über die Dauerhaftigkeit von Legenden und historiographischen Klischees. . . . . . . . . .

Kard Strobel (Heidelberg), Aspekte des politischen und sozialen Scheinbildes der rabbinischen Tradition. Das spätere 2. und das 3. Jh. n. Chr. - Mit einem Anhang: Zur Münzprägung von Diocaesarea-Sepphoris in severischer Zeit . . . . . . . . . . . . . . . . .

MaRGARITA TAČEVa (Sofia), Die politische Dezentralisierung im Odrysenreich vom letzten Viertel des 5. Jh. bis zur Thronbesteigung Kotys' I. 383 v. u. Z.

KLaUs WaChtel (Berlin), War D. Terentius Scaurianus der erste Statthalter Dakiens?. . . . . . . . . . . . . . . 473

Graham J. Wylte (Melbourne), Pompey megalopsychos. . . . . . . . . . . 445

\section{Literaturkritik}

Rezensionen

GERT AUdRING (Berlin): Sozialmaßnahmen und Fürsorge, hrsg. von Hans Kloft, Graz 1988 . . . . . . . . . . . . . . . 277

Azat Bozoyan (Jerewan) - HaNs Ditrten (Berlin): A. A. Akopjan, Albanija Aluank v greko-latinskich i drevnearmjanskich istočnikach, Erevan 1987

GeRdA von BüLow (Berlin): José Dörig, Les trésors d'orfèvrerie thrace. . . .

GeRDA von BüLOW (Berlin): Gunther Gottlieb, Das römische Augsburg, München $1981 \ldots \ldots 32$ 
Hans Ditrex (Berlin): Phaedon Malingoudis, Slawen im mittelalterlichen Griechenland, Thessalonike 1988 (griech.). . . . . . . . . . . . . . . . . 341

Hans Ditten (Berlin): Thérèse Olajos, Les sources de Théophylacte Simocatta historien . . . . . . . . . . . . . . . . . . . . . .

Bernhard Döhle (Berlin): C. Scorpan, Limes Scythicus, Oxford 1980; I. B. Cătăniciu, Evolution of the System of Defence Work in Roman Dacia, Oxford 1981. . . . . . . . . . . . . . . . . . . . . . . .

Teja Ers (Berlin) : Burkhard Rode - Siegrid Weber, Zur Entstehung des Feudalismus in Europa . . . . . . . . . . . . . . . . . . . . .

HaGen Frscher (Berlin): Leonhard Alexander Burckhardt, Politische Strategien der Optimaten . . . . . . . . . . . . . . . . . . . . .

HaGen Frscher (Berlin): Raphaela Drexhage, Untersuchungen zum römischen Osthandel . . . . . . . . . . . . . . . . . . . . . 592

Hagen Frscher (Berlin): Ursula Ortmann, Cicero, Brutus und Octavian. . 588

Bernd Florath (Berlin): Historische Anthropologie. Der Mensch in der Geschichte, hrsg. von H. Süssmuth, Göttingen 1984 . . . . . . . . . . .

BerND Funck (Berlin): Jacques Duchesne-Guillemin, Iran und Griechenland in der Kommagene, Konstanz 1984 . . . . . . . . . . . . . . . . . .

Bernd Funck (Berlin): Klaus Schippmann, Grundzüge der parthischen Geschichte, Darmstadt 1980. . . . . . . . . . . . . . . . . . . . . .

RIgobert GÜNTher (Leipzig): Autori vari, La pace nel mondo antico, a cura di Marta Sordi, Milano 1985. . . . . . . . . . . . . . . . . . .

Rigobert Günther (Leipzig): Suzanne Teillet, Des Goths à la nation gothique, Paris 1984 . . . . . . . . . . . . . . . . . . . . . 332

Rugobert Günther (Leipzig) : Mario Torelli, Lavinio e Roma, Roma 1984. • 312

KLaUs HaLlof (Berlin): Michael Wörrle, Stadt und Fest im kaiserzeitlichen Kleinasien . . . . . . . . . . . . . . . . . . . 608

Klaus-Peter Johne (Berlin): Urbano Espinosa Ruiz, Debate Agrippa - Mecenas en Dion Cassio, Madrid 1982. . . . . . . . . . . . . . . . .

Renate und Klaus-Peter Johne (Berlin): G. S. Knabe, Drevnij Rim-istorija i porsednernost', Moskra 1986 . . . . . . . . . . . . . . . .

FrITz Jürss (Berlin) : Bernard C. Dietrich, Tradition in Greek Religion, BerlinNew York 1986 . . . . . . . . . . . . . . . . . . . .

FrITz Jürss (Berlin) : Literatur und Philosophie in der Antike, hrsg. von Heikki Koskenniemi - Siegfried Jäkel - Vappu Pyykkö, Turku 1986. . . . . . 275

JaNa Kepartová (Prag) : Filippo Coarelli, Il Foro Boario . . . . . . . . . . 600

JENs KöHN (Berlin) : Mario Bretone, Storia del diritto Romano, Roma-Bari 1987295

Helga Köpsten (Berlin): James Caimi, Burocrazia e diritto nel De Magistratibus di Giovanni Lido, Milano 1984 . . . . . . . . . . . . . . . . . 334

HaNs Krumarey (Berlin): Rosmarie Günther, Frauenarbeit - Frauenbindung 601 
Hans Krummrey (Berlin): Ulla Hälvä-Nyberg, Die Kontraktionen auf den lateinischen Inschriften Roms und Afrikas . . . . . . . . . . . . . . 603

Hans Krummrex (Berlin): Jiro Kajanto, The Latin Cognomina, Roma 1982; Heikki Solin - Olli Salomies, Repertorium nominum gentilium et cognominum Latinorum, Hildesheim - Zürich - New York 1988. . . . . . . . 314

HATS KRUMMREY (Berlin): Montana I, Sofija 1987 . . . . . . . . . . . . 318

Haxs Krummary (Berlin): Römische Inschriften Lateinisch/Deutsch, hrsg. von Leonhard Schumacher . . . . . . . . . . . . . . . . . . 605

Havs Krummar (Berlin) : Eva Margareta Steinby, Indici complementari ai bolli doliari urbani (CIL XV 1), Roma 1987 . . . . . . . . . . . . . . .

RALPH-JohanNes LiLIE (Berlin-West): Friedhelm Winkelmann, Quellenstudien zur herrschenden Klasse von Byzanz im 8. und 9. Jahrhundert, Berlin 1987

Allax A. Lund (München): Griechische und lateinische Quellen zur Frühgeschichte Mitteleuropas bis zur Mitte des 1. Jahrtausends u. Z., hrsg. von Joachim Herrmann, I: Von Homer bis Plutarch (8. Jh. v. u. Z. bis 1. Jh. u. Z.), Berlin 1988. . . . . . . . . . . . . . . . . . . . . . . .

Klaus-Peter Matschke (Leipzig): Hans-Georg Beck - Fabrizio Conca Carolina Cupane, Il romanzo tra cultura latina e cultura Bizantina, Palermo 1986. . . . . . . . . . . . . . . . . . . . . . .

Klaus-Peter Matschke (Leipzig): Byzantinische Bleisiegel in Berlin (West), bearb. von Paul Speck; Varia II. . . . . . . . . . . . . . . . . .

Danuta Musiaf (Torún): Fregellae II: Il santuario di Esculapio, a cura di Filippo Coarelli, Roma 1986. . . . . . . . . . . . . . . . . 362

Hans Neumann (Berlin): Das Reich Urartu, hrsg. von Volkert Haas. • . . . 575

Françors Paschoud (Berlin): Rigobert Günther - Alexander R. Korsunskij, Germanen erobern Rom, Berlin 1986. . . . . . . . . . . . 339

Johaynes Michael Rainer (Graz): Giuliana Lanata, Legislazione e natura nelle novelle Giustineanee, Napoli 1984. . . . . . . . . . . . . . . 308

Johannes Michael Rainer (Graz): Vincenzo Scarano Ussani, L'utilità e la certezza, Milano 1987. . . . . . . . . . . . . . . . . . . . . . . . .

Antony E. Raubitschek (Palo Alto, Cal.): Überlegungen zu einigen Beiträgen der Konferenz „Ideologische und methodologische Grundprobleme der antiken Geschichtsschreibung" (Klio 66 [1984], 333-478). . . . . . . .

BerNhaRd RINK (Berlin): Rudolf Karl Bulin, Untersuchungen zur Politik und Kriegsführung Roms im Osten; Martin Jehne, Der Staat des Dictators Caesar; David F. Epstein, Personal Enmity in Roman Politics. . . . . .

Elisabetri Rohde (Berlin): Lucia Amalia Scatozza Höricht, I vetri Romani di Ercolano, Roma 1986 . . . . . . . . . . . . . . . . .

Detrlef Rössler (Berlin): Anne Laidlaw, The First Style in Pompeii : Painting and Architecture, Roma 1985 . . . . . . . . . . . . . . . . . . . 
Dethef Rössler (Berlin): Girolamo Zampieri, Bronzetti figurati etruschi italici paleoveneti e romani del Museo Civico di Padova, Roma 1986; Gustavo Traversari, La statuaria ellenistica del Museo Archeologico di Venezia, Roma 1986

Peter Schreiner (Köln): Friedhelm Winkelmann - Gudrun Gomolka-Fuchs, Frühbyzantinische Kultur, Leipzig 1987 . . . . . . . . . . . . . . .

Marta SORdi (Mailand): L. I. Sanders, Dionysius I of Syracuse and Greek Tyranny, London - New York - Sydney 1987 . . . . . . . . . . . . . . 300

Kurt Treu (Berlin): The Oxford Companion of Classical Literature . . . . . 566

KURT TREU (Berlin): Eric G. Turner, Greek Manuscripts of the Ancient World; Guglielmo Cavallo - Herwig Maehler, Greek Bookhands of the Early Byzantine Period . . . . . . . . . . . . . . . . . . . . . .

Cammla Warnke (Berlin): John P. Anton, Aristotle's Theory of Contrarity (Repr.). . . . . . . . . . . . . . . . . . . . . .

Caxulla Warnke (Berlin): Johannes Hahn, Der Philosoph und die Gesellschaft . . . . . . . . . . . . . . . . . . . . .

Frtedhelm Winkelmane (Berlin): Monika Gödecke, Geschichte als Mythos, Frankfurt/Main - Bern - New York - Paris 1987.

Frtedhelm Whrkelmand (Berlin): Alois Grillmeier, Jesus der Christus im Glauben der Kirche, II/1: Das Konzil von Chalkedon (451), Freiburg/Br. - Basel - Wien 1986. . . . . . . . . . . . . . . . . . . . . . . . . .

Friedhelm Wirkelimane (Berlin): Richard P. C. Hanson, Studies in Christian Antiquity, Edinburgh 1985 . . . . . . . . . . . . . . . .

Friedhela Winkelmann (Berlin): Manfred Jacobs, Die Reichskirche und ihre Dogmen, Göttingen 1987 .

Frifdhelm Wrikelmand (Berlin): Edgar Pack, Städte und Steuern in der Politik Julians, Bruxelles 1986 . . . . . . . . . . . . . . . . . . . .

Friedhelm Winkelmans (Berlin): Tullio Spagnuolo Vigorita, Exsecranda pernicies, Napoli 1984. . . . . . . . . . . . . . . . . . . . .

Annotationen

Wolfram Brandes (Berlin): F. Beltrán Lloris - F. Marco Simón, Atlas de Historia Antiqua, Zaragoza 1987 . . . . . . . . . . . . . . . . . . . . .

Haws Ditten (Berlin): A. A. Bozoyan, Byuzandiayi arevelyan k'ałak 'akanut 'yunə ev Kilikyan Hayastanə žb dari 30-70-akan t'vakannerin, Erevan 1988. . . . . . . . . . . . . . . . . . . . 373

RIgobert GÜNTher (Leipzig): Pio Grattarola, Un libello antiaugusteo. . . . 633

Rrgobert Günther (Leipzig): Paolo Martino, Arbiter . . . . . . . . . . . 633 
RIGOBERT GÜNTHER (Leipzig): Marta Sordi, Il mito troiano e l'eredità etrusca di Roma . . . . . . . . . . . . . . 632

Klats HadloF (Berlin): Biagio Virgilio, Epigrafia e storiografia. Studi di Storia Antica I, Pisa 1988 . . . . . . . . . . . . . . . . . . 370

SLAwIK Hedeler (Berlin): Familie, Staat und Gesellschaftsformation. Grundprobleme vorkapitalistischer Epochen . . . . . . . . . . 629

WolfGang Huschiner (Berlin): Jörg Jarnut, Agilolfingerstudien, Stuttgart 1986. . . . . . . . . . . . . . . 371

HaNs Krummrey (Berlin): Monique Dondin-Payre, Un siècle d'épigraphie classique . . . . . . . . . . . . . . . . 636

DetLef Lotze (Jena): Josiah Ober, Fortress Attica . . . . . . . . . . . . 630

Simona Prellemr (Berlin): François Vannier, Finances publiques et richesses privées . . . . . . . . . . . . . . . . 631

HaNs GeORG ThüMmel (Greifswald): Luca Bianchi, Le stele funerarie della Dacia, Roma 1985 . . . . . . . . . . . . 372

KuRt TReu (Berlin): Stefan Merkle, Die Ephemeris belli Troiani des Dictys von Kreta .................. 635

URSula Treu (Berlin) : Ernst Künzl, Der römische Triumph . . . . . . . . 634 
AKADEMIE DER WISSENSCHAFTEN DER DDR ZENTRALINSTITUT

FÜR ALTE GESCHIGHTE UND ARCHÄOLOGIE

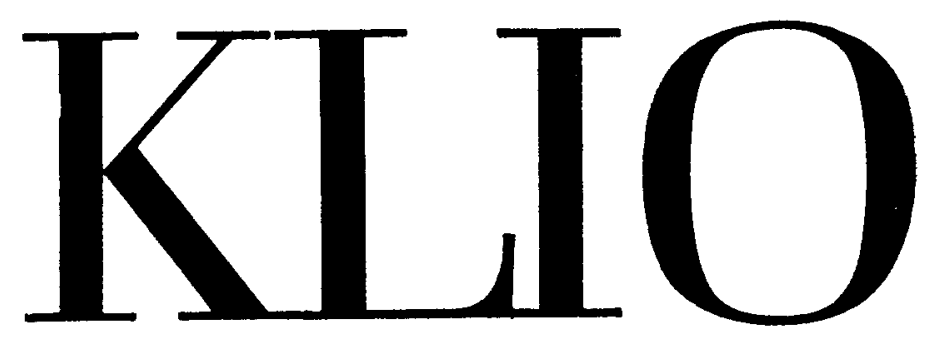

Beiträge zur Alten Geschichte

Band $72 \cdot 1990 \cdot$ Heft 1

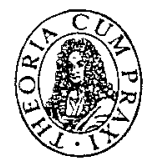

AKADEMIE-VERLAG BERLIN 


\section{REDAKTIONSBEIRAT}

Barry Baldwin (Calgary), Izabella Bieżuńska-Małowist (Warszawa), José M. Blázquez Martinez (Madrid), Janko Fischer (Bucuresti), Boris Gerov (Sofija), Elena S. Golubcova (Moskva), János Harmatta (Budapest), Georgios St. Korrés (Athen), Pavel Oliva (Praha), Dionis M. Pippidi (Bucureştj), Marta Sordi (Milano), Velizar Velkov (Sofija), Ingomar Weiler (Graz).

\section{REDAKTIONSKOLLEGIUM}

Gert Audring (Berlin), Gabriele Bockisch (Rostock), Horst Dieter (Potsdam), Rigobert Günther (Leipzig), Werner Hartke (Berlin), Joachim Herrmann (Berlin), Johannes Irmscher (Berlin), Reimar Mfüller (Berlin).

Verantwortlicher Redakteur: Gert Audring.

Redaktionssekretäre: Katrin Tafere, Wolfram Brandes, Hans Kunde.

Redaktionsschlu日: 10. 11.1989

Manuskripteinsendungen an folgende Adresse:

Akademie der Wissenschaften der DDR, ZIAGA, Redaktion der "Klio“,

Lejpziger Str. 3-4, Postfach-Nr. 1310, DDR - 1086 Berlin.

\section{Bezugsmöglichkeiten}

Bestellungen sind zu richten

- in der $D D R$ an das Zeitungsvertriebsamt der Deutschen Post, Abt. Zentralvertrieb Berlin, StraGe der Pariser Kommune 3-4, DDR - 1004 Berlin, oder an den Akademie-Verlag Berlin, Leipziger Straße 3-4, Postfach-Nr. 1233, DDR - 1086 Berlin;

- in der BRD und Berlin (West) an eine Buchhandlung oder die Auslieferungsstelle KUNST UND WISSEN, Erich Bieber GmbH. Ein Unternehmen der Faxon-Gruppe, Postfach 102844, D - 7000 Stuttgart 10;

- in den übrigen Ländern an den internationalen Buch- und Zeitschriftenhandel bzw. an die Auslieferungsstelle KUNST UND WISSEN, Erich Bieber GmbH. Ein Unternehmen der Faxon-Gruppe, Stampfenbachstr. 73, Postfach, CH • $\mathbf{S 0 3 5}$ Zürich, an den zuständigen Postzeitungsvertrieb des Landes bzw. an den Akademie-Verlag Berlin, Leipziger Str. 3-4, DDR - 1086 Berlin.

Zeitschrift „Klio“

Herausgeber: Zentralinstitut für Alte Geschichte und Archäologie der Akademie der Wissenschaften der Deutschen Demokratischen Republik.

Verantwortlicher Redakteur: Prof. Dr. sc. Gert Audrjng.

Anschrift der Redaktion: Zentralinstitut für Alte Geschichte und Archäologie, Leipziger Straße 3-4, Postfach-Nr. 1310, DDR - 1086 Berlin.

Veröffentlicht unter der Registriernummer 1309.

Veriag: Akademie-Verlag Berlio, Leipziger Str. 3-4, Postfach-Nr. 1233, DDR - 1086 Berlin; Fernruf: 2236201 oder 22362 29; Telex-Nr.: 1144 20; Bank: Staatsbank der DDR, Berlio, Konto-Nr.: 6836-26-20712.

Stellv. Cheflektor Zeitschriften: Armin Beck. Redakteur der Abt. Zeitschriften: Christina Gericke.

Gesamtherstellung: IV/2/14 Druckerei „Gottfried Wilhelm Leibniz“, DDR - 4150 Gräfenhainichen.

Erscheinungsweise: Die Zeitschrift „Klio" erscheint jährlich in einem Band mit 2 Heften. Bezugspre is eines Bandes 160,-DM zuzüglich Versandspesen; Preis je Heft $80,-D M$.

Bestellnummer des Bandes: 1046/72/1.

Urheberrecht: Alle Rechte vorbehalten, insbesondere die der Übersetzangen. Kein Teil dieser Zeitschrift darf in irgendeiner Form - durch Fotokopie, Mikrofilm oder irgendein anderes Verfahren - ohne schriftliche Genehmigung des Verlages reproduziert oder in eine von Maschinen, insbesondere von Datenverarbeitungsanlagen verwendbare Sprache übertragen oder ubersetzt werden. - All rights reserved (including those of translation into foreign languages). No part of this issue may be reproduced in any form, by photoprint, microfilm, or any other means, nor transmitted or translated into a machine language, without written permissions from the publishers.

(c) 1990 by Akademie-Verlag Berlin. Printed in the German Democratic Republic.

AN (EDV) $60913 \cdot$ AN (ZV) 1118001737 